\title{
A importância do Médico Veterinário na Saúde Pública
}

\author{
The importance of the Veterinarian in Public Health \\ La importancia del Veterinario en la Salud Pública
}

Recebido: 16/06/2021 | Revisado: 21/06/2021 | Aceito: 24/06/2021 | Publicado: 10/07/2021

\author{
Adam Rafael Souza dos Anjos \\ ORCID: http://orcid.org/0000-0003-1948-6293 \\ Universidade Federal de Alagoas, Brasil \\ E-mail: heman_rafaell@outlook.com \\ Cícero Taylson Oliveira Alves \\ ORCID: https://orcid.org/0000-0001-6298-6256 \\ Universidade Federal de Alagoas, Brasil \\ E-mail: taylsonalves6@gmail.com \\ Valério Areolino de Souza Neto \\ ORCID: https://orcid.org/0000-0001-5984-0459 \\ Universidade Federal de Alagoas, Brasil \\ E-mail: valerioramosdm11@gmail.com \\ Wagner Richard Alves dos Santos \\ ORCID: https://orcid.org/0000-0002-0846-0891 \\ Universidade Federal de Alagoas, Brasil \\ E-mail:wagner.santos@arapiraca.ufal.br \\ Denise Maria Santos \\ ORCID: https://orcid.org/0000-0001-8886-6439 \\ Instituto Dom José de Educação e Cultura, Brasil \\ E-mail: deniseufc@yahoo.com.br \\ Maria José de Holanda Leite \\ ORCID: https://orcid.org/0000-0003-4154-3901 \\ Universidade Federal de Alagoas, Brasil \\ E-mail: maryholanda@gmail.com
}

\section{Resumo}

Foi a partir da resolução no 287 de 08 de outubro de 1998 do Conselho Nacional de Saúde - CNS que os médicos veterinários passaram a ser considerados profissionais da saúde, e, desde então, é possível notar a expansão do campo de atuação destes profissionais e a sua importância no que tange à saúde pública, haja vista, 60\% das doenças humanas são de caráter zoonótico e $75 \%$ dessas doenças têm origem na fauna silvestre, e os únicos profissionais habilitados para prevenir, diagnosticar e tratar esses ricos em animais e evitar que cheguem aos humanos é o profissional da medicina veterinária. O objetivo do estudo foi descrever a formação cada vez mais holística dos médicos-veterinários, sua atuação nas diferentes áreas da saúde pública brasileira e a importância deles para a saúde única - interface entre a saúde animal, humana e ambiental. Foi utilizada uma revisão de literatura por meio de coleta de dados em revistas, sites, artigos e periódicos. A pesquisa resultou em uma riqueza de informações muito variadas sobre alguns campos de atuação dos médicos veterinários na saúde coletiva, e levantou importantes debates sobre o que será que ainda leva alguns gestores de saúde a resistirem na contratação deste profissional, tendo em vista que no ano de 2018 dentre os 5.570 municípios brasileiros somente 48 destes possuíam o médico-veterinário nas equipes de Núcleo Ampliado de Saúde da Família - NASF, colocando em questão seu relevante papel destes para a preservação e promoção da saúde humana, e não somente animal.

Palavras-chave: Medicina veterinária preventiva; Zoonoses; Saúde única; NASF; Vigilância sanitária e ambiental.

\begin{abstract}
It was from Resolution No. 287 of October 8, 1998 of the National Health Council - CNS that veterinarians started to be considered health professionals, and, since then, it is possible to notice the expansion of the field of action of these professionals and the its importance with regard to public health, given that $60 \%$ of human diseases are zoonotic and $75 \%$ of these diseases originate in wild fauna, and the only professionals qualified to prevent, diagnose and treat these rich in animals and prevent reach humans is the veterinary medicine professional. The aim of the study was to describe the increasingly holistic training of veterinarians, their performance in different areas of Brazilian public health and their importance for unique health - the interface between animal, human and environmental health. A literature review was used through data collection in journals, websites, articles and journals. The research resulted in a wealth of very varied information about some fields of activity of veterinarians in public health, and raised important debates about what still leads some health managers to resist hiring this professional, considering that in the year of 2018, among the 5,570 Brazilian municipalities, only 48 of these had the veterinarian in the teams of the
\end{abstract}


Expanded Family Health Center - NASF, calling into question their relevant role in the preservation and promotion of human health, and not just animal health.

Keywords: Preventive veterinary medicine; Zoonoses; Unique health; NASF; Health and environmental surveillance.

\section{Resumen}

Fue a partir de la Resolución N ${ }^{\circ} 287$ del 8 de octubre de 1998 del Consejo Nacional de Salud - CNS que los veterinarios pasaron a ser considerados profesionales de la salud y, desde entonces, es posible notar la ampliación del campo de acción de estos profesionales y el su importancia en lo que respecta a la salud pública, dado que el $60 \%$ de las enfermedades humanas son zoonóticas y el $75 \%$ de estas enfermedades se originan en la fauna silvestre, y los únicos profesionales capacitados para prevenir, diagnosticar y tratar estos ricos en animales y evitar que lleguen a los humanos es el veterinario. profesional de la medicina. El objetivo del estudio fue describir la formación cada vez más holística de los veterinarios, su desempeño en diferentes áreas de la salud pública brasileña y su importancia para la salud única: la interfaz entre la salud animal, humana y ambiental. Se utilizó una revisión de la literatura a través de la recolección de datos en revistas, sitios web, artículos y revistas. La investigación arrojó un caudal de información muy variada sobre algunos campos de actividad de los veterinarios en salud pública, y suscitó importantes debates sobre lo que aún lleva a algunos gestores sanitarios a resistirse a contratar a este profesional, considerando que en el año de 2018, entre los 5.570 brasileños municipios, solo 48 de estos contaban con el veterinario en los equipos del Centro Ampliado de Salud Familiar - NASF, cuestionando su papel relevante en la preservación y promoción de la salud humana, y no solo la salud animal.

Palabras clave: Medicina veterinaria preventiva; Zoonosis; Salud única; NASF; Vigilancia sanitaria y ambiental.

\section{Introdução}

Segundo a Organização Mundial de Saúde - OMS, Saúde é o completo bem-estar, físico, mental, social e espiritual e não somente a ausência de doença. (OMS, 1946). Logo, compreende-se como saúde pública, todo o conjunto de medidas executadas pelo Estado - União, Estados, Distrito Federal e municípios - para garantir o bem-estar geral da população. Para que isso seja possível, os seres humanos consigam alcançar o bem-estar, definido pela OMS, é necessário que alguns fatores estejam em equilíbrio, como revelou o psicólogo humanista Maslow, no ano de 1954, por meio da obra "A Theory of Human Motivation".

A saúde pública veterinária tem a finalidade de proteger, prevenir e promover a saúde humana, através dos cuidados preventivos com os seus clientes animais, por meio da vacinação, avaliação nutricional, exames anuais, no rastreamento e manejo adequado de patógenos com potencial zoonótico. A partir da Portaria 2.488 de outubro de 2011 a medicina veterinária de nível superior foi incluída no grupo de profissionais que podem compor o Núcleo Ampliado de Saúde da Família - NASF (Begali, 2015). E, desde então, o campo de atuação para este profissional dentro da saúde pública passou a ampliar-se cada vez mais.

Nos dias atuais é possível encontrar veterinários exercendo relevantes contribuições no cenário da saúde coletiva, como: nas inspeções de alimentos de origem animal em abatedouros, frigoríficos e supermercados; na vigilância sanitária e ambiental, comandando ações de combate às arboviroses, a exemplo da dengue - doença de ciclos endêmicos e epidêmicos pelo seu comportamento sazonal no Brasil; em alguns Estados compondo as equipes do NASF, na realizando visitas domiciliares e atividades de educação em saúde às comunidades; e nos centro de controle de zoonoses - CCZ, realizando o rastreamento e combate das doenças com potenciais zoonóticos, como é o caso da Leishmaniose, doença que acomete animais e humanos. (Menezes, 2005).

O médico-veterinário também cuida do ser humano! Nem todo mundo sabe, mas $62 \%$ dos patógenos humanos conhecidos são transmitidos por animais (Cunningham, 2005), além disso, 75\% das doenças emergentes tiveram origem na fauna silvestre (Blancou et al., 2005). Essas pesquisas comprovam o quanto é importante a participação efetiva deste profissional na saúde pública, pois, dentre os profissionais da saúde, os veterinários são os únicos qualificados cientificamente para lidar com as questões de saúde animal. Usando-se da prevenção, manejo correto, rastreamento de novos agravos e controlando as afecções e infecções em seus pacientes, evitar-se-á que novas pandemias cheguem a ocorrer como é o caso da 
COVID-19, que já matou mais de 440 mil brasileiros até o dia 20/05/2021, segundo divulgação do centro de estudos em COVID da Universidade Johns Hopkins, situada nos Estados Unidos.

Desse modo, fica claro que os profissionais que antes eram vistos somente como sendo da área das ciências agrárias, e na visão popular, responsáveis somente pela saúde dos animais domésticos, desde 1998 passam a pertencer à categoria de profissional de saúde, reconhecidos pelo Conselho Nacional de Saúde (CNS) (CFMV, 2015). De acordo com a Lei ${ }^{\circ}$ 5517/1968, o estudo e a aplicação de medidas de saúde pública no tocante às doenças de animais transmissíveis ao homem, as zoonoses, é uma das funções do médico-veterinário.

Tendo em vista a ampliação do campo de atuação dos profissionais médicos veterinários, o Conselho Federal de Medicina Veterinária e suas Regionais em parceria com o Ministério da Educação - MEC instituiu a complementaridade de disciplinas consideradas relevantes para a formação acadêmica destes, por meio da resolução no 3 , de 15 de agosto de 2019 , que estabelece as diretrizes curriculares nacionais do curso de graduação em medicina veterinária, ratificando o compromisso com a formação ainda mais holística destes futuros profissionais, reafirmando às exigências cada vez mais crescentes do mercado no tocante à saúde única - interface da saúde humana; animal e ambiental -, termo este definido pelo médico veterinário norte-americano Calvin W. Schwabe (1927-2006) na obra "Veterinary Medicine and Human Health" publicada em 1984, e que passou a ser tão utilizado nas discussões científicas que tratam de questões ligadas à epidemiologia.

O presente texto tem como objetivo fornecer informações sobre: importância do médico veterinária no campo da saúde pública e a sua atuação e contribuição na prevenção de doenças de caráter zoonótico na saúde humana e a redução dos potenciais riscos para o surgimento de novas pandemias.

Portanto, por se tratar de uma revisão de literatura, usou-se como metodologia de pesquisa: artigos, revistas acadêmicas, (sites Google Acadêmico, SciELO e Periódico - portal da CAPES), escolheu-se artigos e documentos específicos voltados aos estudos da importância dos profissionais veterinários e sua contribuição para a saúde pública.

\section{Formação Acadêmica do Profissional Médico Veterinário}

Atividades como cuidar da saúde dos animais, diagnosticarem doenças animais, prescrever medicações de forma segura e consciente, são tarefas exclusivas do médico-veterinário, entre essas atividades as cirurgias e clínica médica de animais. O que poucos sabem é que o médico-veterinário desenvolve um papel importante e fundamental na saúde pública, mesmo sendo pouco conhecido nessa área o estudante de Medicina Veterinária sai da universidade apto a atuar na saúde pública e até coordenar equipes de vigilância sanitária. Isso graças à grade curricular desse profissional que ao passar do tempo foi sendo modificada, permitindo sua atuação diretamente na gestão da saúde.

Este profissional já vem utilizando seus conhecimentos de biologia, ecologia, medidas gerais de profilaxia, medicina veterinária preventiva, administração, entre outros, para desempenhar várias funções nas diferentes áreas da Saúde Pública, inclusive coordenando as equipes de vigilância (Cleiton, 2005). Isso se tornou possível devido às mudanças curriculares que permitiram durante a graduação ganhar a oportunidade de mergulhar em conhecimentos específicos voltados para a área da saúde, complementando sua formação profissional. Assim argumenta Cicero Cleiton (2005). Os cursos superiores têm dado ao ensino teórico e prático dos conteúdos relacionados às diferentes áreas de atuação do médico-veterinário na Saúde Pública.

Devido ao aperfeiçoamento das grades curriculares, se expandiu as áreas de atuação e cresceu as habilidades de trabalho na profissão. Isso porque durante a formação profissional, os estudantes conhecem e praticam disciplinas importantes que irão capacitá-los a exercer atividades como; o controle de zoonoses e a segurança sanitária dos produtos de origem animal. Disciplinas como: Epidemiologia e Saúde Pública, Higiene Veterinária, Defesa Sanitária Animal e Zoonoses, Inspeção de Produtos de Origem Animal e Ecologia. Áreas de aprendizados que permite formar médicos-veterinários preparados para 
cuidar também da saúde das pessoas. Tem sempre um médico-veterinário cuidando dos animais, do ambiente e de você. (Autran, 2020).

Como já visado, o médico-veterinário é fundamental na saúde pública, ainda que um pouco desvalorizado pela maioria das pessoas e dos governos, mesmo sua importância na área da saúde foi reconhecida pela Organização Mundial da Saúde - OMS desde 1975. Foi um passo importante para a formação dos médicos-veterinários, mas não o ideal. A maioria dos cursos de Medicina Veterinária não possui uma estrutura favorável ao desenvolvimento de atividades práticas relacionadas às áreas de atuação do médico-veterinário na saúde pública (Cleiton, 2005) e mesmo com todo esse processo de reconhecimento que a medicina veterinária vem passando ainda falta muito a trilhar e a lutar por mais valorização dos médicos-veterinários na saúde pública e incentivo aos alunos a procurar mais essa área da saúde tão importante.

Por outro lado, é bem observado que mesmo com disciplinas tão focadas a preparar os alunos a serem profissionais capazes de atuar na saúde coletiva, muitas das universidades não possuem professores com experiência nessa área, deixando assim o estudo muito concentrado só na parte teórica.

Como contribuição da formação acadêmica, se preocupando em formar profissionais preparados a evitar o surgimento de novas zoonoses, doenças que venha a atingir a população, enfermidades essas de origem animal seja doméstico ou silvestre, os governos tanto municipais e estaduais deveriam criar programas de estágios nas secretárias de saúde, criando oportunidades de alunos a estagiarem em Unidades Básicas de Saúde. Atualmente, algumas prefeituras de Pernambuco estão oferecendo estágios inclusive remunerados, nos serviços de Vigilância Sanitária aos estudantes da UFRPE (Cleiton, 2005). Isso enriquece muito a formação dos alunos dos cursos de Medicina Veterinária, além de aumentar o desejo estudantes de atuar nessa área importante e fundamental para a saúde das pessoas.

\section{O Surgimento das Atividades de Medicina Veterinária Preventiva}

Este período tem seu princípio na pré-história e continua até o primeiro século da era cristã. O código de Hamurabi, $1^{\circ}$ código de leis mais antigo do mundo, já havia menções de práticas de medicina veterinária, onde havia indivíduos que tinha essa atribuição, vemos que essa profissão é mais antiga do que se tem conhecimento. Os primeiros esforços dirigidos contra a doença animal que se tem conhecimento foram descritos nas antigas civilizações da Suméria, Egito e Grécia, com referências a curandeiros de animais antes da era cristã.

Ao lado do tratamento médico, cirúrgico e obstétrico individual, duas outras táticas eram aplicadas localmente para o controle das enfermidades animais, antes ainda que tivesse sido desenvolvida a teoria do contágio: o emprego da quarentena (segregação dos animais doentes dos sadios) e o sacrifício de animais enfermos.

\subsection{Cenário atual e tendências da medicina veterinária preventiva e saúde pública}

Hoje em dia a um foco muito grande no que diz respeito a medicina veterinária na saúde humana e do controle de qualidade dos alimentos, e uma política muito tímida na saúde dos animais, este último deve estar na pauta das políticas tendo em vista que ,para muitos proprietários de animais os mesmos são como filhos e as pessoas gostam de dar o tratamento adequado para seus animais, porém ,nas clínicas particulares cobram caro e muitas vezes os donos dos pets tem que escolher entre comer, pagar as contas ou levar seu bicho a uma clínica.

Sendo assim, o governo deve criar um hospital veterinário gratuito, centralizado nas cidades e que atenda a população mais pobre que não tem condições de levar seus animais a uma clínica privada. 
A expressão saúde pública veterinária é utilizada para designar o marco conceitual e a estrutura de implementação das atividades de saúde pública que empregam conhecimentos e recursos da medicina veterinária para proteger e melhorar a saúde humana. A saúde pública veterinária vincula a agricultura, a saúde animal, a educação, o ambiente e a saúde humana.

$\mathrm{O}$ autor argumenta que em um mundo com uma população cada vez mais numerosa, que recorre a novos sistemas de exploração do solo e a novas tecnologias, é importante o desenvolvimento de uma medicina veterinária populacional. A orientação dispensada à medicina veterinária dentro da tríade formada pelo meio ambiente, o animal e o homem devem ser acompanhados de uma importante expansão da saúde pública veterinária.

Tradicionalmente dentro do âmbito da saúde pública, a medicina veterinária tem trabalhado no controle das zoonoses e na proteção sanitária dos alimentos. Para realizar atividades ligadas à área ambiental, Cifuentes (1992) aponta que o médico veterinário deve ter conhecimentos gerais sobre as ciências do ambiente, além de conhecimentos sobre: a) as relações ambiente-enfermidade; b) as atividades agropecuárias e suas relações sobre o ambiente; c) modelos de avaliação de estudos de impacto ambiental; d) tecnologia básica para a proteção e saneamento ambiental. Na formação acadêmica dos médicos veterinários, o autor propõe que as escolas ofereçam conhecimentos aprofundados nas áreas de ciências ambientais, ecologia, biologia e saneamento ambiental para que os profissionais possam ser incorporados e oferecer sua contribuição a esses setores.

Para realizar atividades ligadas à área ambiental, Cifuentes (1992) aponta que o médico veterinário deve ter conhecimentos gerais sobre as ciências do ambiente, além de conhecimentos sobre: a) as relações ambiente-enfermidade; b) as atividades agropecuárias e suas relações sobre o ambiente; c) modelos de avaliação de estudos de impacto ambiental; d) tecnologia básica para a proteção e saneamento ambiental. Na formação acadêmica dos médicos veterinários, o autor propõe que as escolas ofereçam conhecimentos aprofundados nas áreas de ciências ambientais, ecologia, biologia e saneamento ambiental para que os profissionais possam ser incorporados e oferecer sua contribuição a esses setores. Nielsen (1997) declara que o profissional de Medicina Veterinária deve ter um nível de competência consistente com as demandas da sociedade.

O problema das zoonoses é destacado por vários autores, esse grupo de enfermidades continua a representar um importante problema de saúde para grande parte do mundo, com elevadas perdas para os setores de saúde e de agricultura, principalmente nos países em desenvolvimento. O risco de infecções emergentes por novas entidades patológicas ou por agentes conhecidos aparecendo em novas áreas ou em novas condições vem aumentando nos últimos anos.

\subsection{O médico veterinário na vigilância sanitária}

A Vigilância Sanitária (VS) é destinada à defesa da saúde pública, promovendo um domínio sanitário de produção e comercialização (ANVISA), onde atua contra os efeitos contraproducentes gerados por motivos de alguma distorção sanitária. Existe uma variedade de profissionais sanitaristas, entre eles o Médico Veterinário, sendo responsável pela higienização e distribuição de produtos de origem animal.

A VS deve atuar para fiscalizar e proteger a saúde da população, diminuindo ou impedindo possíveis riscos a saúde e solucionar problemas sanitários decorrentes do meio ambiente, da produção, da circulação de bens de interesse da saúde populacional. É dever de o Estado realizar a vigilância e controle dos produtos para promoção da saúde, deste modo o Governo Federal criou a ANVISA Agência Nacional de Vigilância Sanitária, através da lei nº 9.782/99, definindo o Sistema Nacional de Vigilância Sanitária (SNVS), o qual é instrumento que o Sistema Único de Saúde dispõe para realizar seus objetivos de prevenção, controle e promoção da saúde.

Dentre os profissionais de saúde que atuam na Vigilância Sanitária, o Médico Veterinário tem uma função vital. É atribuição do Médico Veterinário, fiscalizar todos os produtos de origem animal, atuando no controle de enfermidades que podem ser transmitidas através de produtos processados de forma inadequada, contendo patógenos e também como doenças de riscos zoonóticos para a população, muitas dessas podendo ser letais e de fácil disseminação. Além disso, o Médico 
Veterinário é o único profissional com capacidade de atuar como sentinela contra doenças estabelecidas e doenças emergentes que possam acometer animais e trazer grandes riscos para a saúde humana.

O médico veterinário exerce a função de fiscalização dando ênfase a higienização e sanidade de produtos de origem animal. Assim evitando a propagação de doenças com grande poder de difusão, que poderá impor restrições ao comercio de animais e derivados de origem, causando danos à saúde pública (BORGES, 2010), onde é o caso das zoonoses que ainda acometem um grande número de animais e humanos. Os casos mais populares dessas doenças são: a tuberculose bovina causada pela Mycobacterium bovis, e a triquinose e yersiniose que acometem suínos, e podem ser transmitidas ao homem, em casos de não inspeção da carne antes do consumo.

A segurança alimentar sanitária dos alimentos de origem animal é uma questão relevante, constituindo uma exigência da sociedade, sendo um tema de interesse direto do médico veterinário que além do encargo legal deve alargar as suas competências para participar com efetividade desta importante área de atuação profissional. A inocuidade sanitária é preocupação de todos, onde casos de doenças provocadas por ingestão de produtos de origem animal contaminados podem vim a se tornarem pandemias (OMS), além de trazerem dúvidas e preocupação ao mercado e principalmente ao consumidor.

O médico veterinário é um profissional indispensável para assegurar a saúde da população humana e animal, e o seu trabalho é fundamental para o desenvolvimento da humanidade no aspecto cientifico, nutricional, sanitário e em perfeito equilíbrio com o meio ambiente e com os animais que aqui habitam.

O médico veterinário tem sua importante função na saúde humana, em parceria com a vigilância sanitária o médico veterinário é responsável por intervir e averiguar a condição de cada produto que vem a depender do animal, como por exemplo a carne bovina. Outra grande função é na prevenção de doenças que podem ser simples ou até mesmo catastrófica ao ponto de virar uma pandemia, como a do Sars-CoV-2. A vigilância sanitária é o órgão responsável por inspecionar os alimentos com destino aos humanos, com isso o médico veterinário ganhou um grande espaço nesse órgão, o rebanho destinado ao consumo de carne aos humanos vem sendo acompanhado pelo médico veterinário desde bezerro a fase fina de abate, para averiguar a qualidade da carne, saber se está apropriada ao consumo humano ou não. A maioria das doenças tem tido origem em animais, como por exemplo; a grande pandemia do século XXI, COVID-19; a mesma teve início na china, em uma feira ao ar livre, onde um chinês consumiu a carne de um animal infectado por um determinado vírus, o alimento ingerido não passou por uma inspeção e com isso o humano que consumiu se infectou com a doença e saiu espalhando para a população em volta, até se tornar o colapso que há hoje.

Portanto, hoje sabemos a verdadeira e importante função do médico veterinário na vigilância sanitária, o papel responsável por prevenir e cuidar de vidas, porém está faltando o seu devido espaço na sociedade para o médico veterinário, infelizmente a sociedade não valoriza de forma certa esse importante profissional.

\subsection{O médico veterinário no Núcleo Ampliado de Saúde da Família e Atenção Básica (NASF-AB)}

O Núcleo Ampliado de Saúde da Família e Atenção Básica (NASF-AB) foi criado pelo Ministério da Saúde, por meio da portaria $\mathrm{n}^{\circ} 154$, de 24 de janeiro de 2008, com o objetivo de apoiar a consolidação das ações prestadas pelas equipes de Saúde da Família - eSFs, aos usuários do Sistema Único de Saúde, atuando de forma complementar nas atividades de educação em saúde e assistência preventiva. Em cada esfera municipal que possuem as suas eSFs, deve constar a presença de uma equipe de NASF-AB, que servirá de suporte para todas as equipes da atenção básica. (CFMV, 2018).

O NASF é composto por uma equipe multiprofissional que desempenha assistência multidisciplinar, de forma integrada, tendo assim, maior capacidade de intervir em problemas, atendendo as necessidades dos municípios em termos sanitários e ambientais (CRMVPB, 2015). Entre os profissionais indicados como possibilidade de membros dessa equipe 
encontram-se o profissional da medicina veterinária, que só em 2011 passou a integrar à lista. Lembrando que, não é obrigatória a convocação de todos os profissionais listados, como deixa bem claro a portaria normativa.

Sendo assim, ficando a encargo de cada gestor municipal, estruturar as suas equipes, pautando-se nos estudos epidemiológicos das suas áreas de saúde. De acordo com o Cadastro Nacional dos Estabelecimentos de Saúde do Brasil (CNES), do Ministério da Saúde, existia até dezembro de 2018 apenas 48 médicos-veterinários atuando no NASF, um número até então pequeno se comparado aos 5.570 municípios da federação e do Distrito Federal.(IBGE, 2016), os números revelam que aproximadamente $0,87 \%$ (zero vírgula oitenta e sete por cento) dos municípios brasileiros reconhecem por meio dos seus gestores, a importância deste profissional para a saúde pública local, relevando ao mesmo tempo a resistência que há ao inserir essa categoria entre os profissionais da saúde.

No estado de São Paulo, que possui 645 municípios, em apenas 16 cidades consta a presença deste nas equipes dos Núcleos. Por mais incrível que pareça, a Capital não faz parte desse rol. (CRMVSP, 2019). Sendo este profissional da saúde, essencial na prevenção e combate as doenças de origem animal. Ainda é nítida a resistência dos gestores municipais prefeitos, e secretários de saúde, na contratação, lotação e consagração de vagas em editais de concursos públicos para ocupação nestes espaços profissionais.

Portanto, a presença dos médicos veterinários no NASF melhora a qualidade da atenção básica à família. São eles que realizam as visitas domiciliares para diagnóstico de risco à saúde na interação entre seres humanos, animais e meio ambiente, como bem destaca o CFMV. Os profissionais de Medicina Veterinária participam do desenvolvimento de ações educativas e de mobilização contínua da comunidade, ajudando no controle de doenças e conscientização sobre o uso e manejo adequado do território. Além disso, participam de estudos e pesquisas em saúde pública, orientam sobre cuidados com manejo de resíduos, previnem e controlam doenças transmissíveis por alimentos, vetores, animais e alterações ambientais provocadas pelo ser humano e desastres naturais.

\section{Considerações Finais}

Como visto, a formação do médico-veterinário vai além da saúde animal, contribuindo também para a saúde humana e do ambiente. Sendo este o profissional altamente habilitado para identificar juntamente com os demais profissionais da saúde, os riscos eminentes a saúde pública e preveni-las de forma correta e sem maiores danos. A formação acadêmica cada vez mais especializada torna o aluno e futuro profissional da medicina veterinária capaz de responder com segurança e de forma precisa as demandas cada vez mais crescente no que tangem os estudos e assistência ao combate e surgimentos de novas pandemias como a do COVID-19 e de outras doenças que venham a se tornar ameaças futuras.

\section{Referências}

Cleiton, C. F. M. Brasil Escola. atuação do médico veterinário na saúde pública. <https://meuartigo.brasilescola.uol.com.br/saude/atuacao-medico-veterinariona-saude-publi ca.htm>

CFMV. O médico-veterinário é profissional de Saúde Pública.01/09/2020 - Atualizado em 02/09/2020.Disponível em: <https://www.cfmv.gov.br/o-medicoveterinario-e-profissional-de-saude

publica/comunicacao/noticias/2020/09/01/\#: :text=De\%20acordo\%20com\%20a\%20Lei,Sa\%C3\%BAde\%20(CNS)\%20desde\%201998>. Acesso em: $20 / 05 / 2021$

CRMVSP. Participação do médico-veterinário é fundamental na garantia da Saúde Única. Disponível em: https://www.crmvsp.gov.br/site/noticia ver.php?idnoticia=7155>. Acesso em: 19/05/2021.

Müller, D.; Antônio MenineVielmo, M.; Carpes Gindri, F.; Maciel Vanhove, R.;HenriqueMattanaPiccoli, V.; Izabel Funguetto, C. Divulgando a Atuação do Médico Veterinário na Vigilância Sanitária. Anais do Salão Internacional de Ensino, Pesquisa e Extensão, v. 10, n. 3, 14 fev. 2020. 
Research, Society and Development, v. 10, n. 8, e18210817254, 2021

(CC BY 4.0) | ISSN 2525-3409 | DOI: http://dx.doi.org/10.33448/rsd-v10i8.17254

Alves C. M.; Lins, G. G. J. Atuação do médico veterinário na vigilância sanitária, Disponível em:

<https://cmfc.emnuvens.com.br/brasileiro/issue/view/3d.> 2013. Acessado em 20/05/2021

CFMV, Revista 84. Desafios da Pandemia.As informações que possibilitaram traçar, mesmo que de forma precária, a origem da pandemia do SARS-CoV-2 e a sua história natural se valeram principalmente de estudos de campo pregressos. Brasília, DF, Ano XXVIDisponívelem: <https://www.cfmv.gov.br/wpcontent/uploads/2020/01/revista84.pdf>. Acesso em: 18/05/2021

Gonçalves, S. R. F.; Silva, Ó. P., Melo, K. M., Brandespim, D. F. O Médico Veterináriono Núcleo Ampliadode Saúdeda Famíliae Atenção Básica (NASF-AB)

IBGE. Número de municípios brasileiros. Disponível em: <https://www.ibge.gov.br/cidades-e-estados.html?view=municipio> Acesso em: 19/05/2021

BRASIL. Ministério da Saúde. Portaria nº 2.488, de 21 de Outubro de 2011. Brasília, DF: MS; 2011. Aprova a Política Nacional de Atenção Básica, estabelecendo a revisão de diretrizes e normas para a organização da Atenção Básica, para a Estratégia Saúde da Família (ESF) e O Médico Veterinário no Núcleo Ampliado de Saúde da Família e Atenção Básica (Nasf-AB) Rev. Ciên. Vet. Saúde Públ., v.6, n. 2, p. 388-396, 2019Programa de Agentes Comunitários de Saúde (PACS). Diário Oficial da União. 21 Out 2011.

IEpifânio, I. S , Brandespim, D. F. Contribuição do Médico Veterinário na Atenção Primária à Saúde: Um Relato de Experiência, ARS Veterinaria, Jaboticabal, SP, v.35, n.2, 050-055, 2019 